\title{
Complete genome analysis of enterotoxigenic Escherichia coli K88 phage vB_EcoP_E212, a novel genus Lederbergvirus
}

\section{Bingdong Wei}

Jilin Academy of Agricultural Sciences

\section{Cong Cong}

Dalian University of Technology

\section{Lin Zheng}

Jilin Academy of Agricultural Sciences

\section{Long Chen}

Jilin Academy of Agricultural Sciences

Xiaogang Yan ( $\nabla$ yanxiaogang1977@163.com )

Jilin Academy of Agricultural Sciences https://orcid.org/0000-0003-0234-6964

\section{Research Article}

Keywords: enterotoxigenic Escherichia coli K88, phage, genome analysis, biological characteristics

Posted Date: September 24th, 2021

DOl: https://doi.org/10.21203/rs.3.rs-852746/v1

License: (c) (i) This work is licensed under a Creative Commons Attribution 4.0 International License. Read Full License 


\section{Abstract}

The features and genome annotation of a newly bacteriophage v B_EcoP_E212 (referred to as E212) which isolated from farm sewage collected in Jilin, China was describes in this study. Bacteriophage E212 belongs to the family Podoviridae, order Caudovirales through transmission electron microscopy. This phage specifically infects enterotoxigenic Escherichia coli K88. The dsDNA molecule of phage E212 was 38252 bp in length and contained $46.98 \% \mathrm{G}+\mathrm{C}$ content. It has been predicted to contain $53 \mathrm{ORFs}$, and no tRNAs. Phage E212 carried the integrase gene, and no homologues of virulence factors or antimicrobial resistance genes were found in this phage. Phage E212 was assigned to the genus Lederbergvirus in accordance with nucleotide sequence alignment and phylogenetic analysis.

\section{Introduction}

Diarrheal disease due to enterotoxigenic Escherichia coli (ETEC) is the most common enteric colibacillosis encountered in neonatal and early-weaned piglets [1]. The main virulence attributes of ETEC are adhesins (fimbriae) and enterotoxins (proteins) [2], both essential for disease development. Among the different ETEC, including K88 (F4), K99 (F5), 987p (F6), F41and F18, the K88 or F18 are the most prevalent form of $E$. coli infections [3]. E. coli colonizes the small intestine via fimbrial adhesins that bind to glycoprotein receptors on the enterocyte brush border and subsequently secretes heat-labile enterotoxins and/or heat-stable enterotoxins that cause diarrhea by altering tight junction integrity and disrupting paracellular passages for ions, solutes and water [4]. Treatment of ETEC infection could be common by the administration of antibiotics, but antibiotic abuse may lead to drug residues [5], immunity decline in animals [6], bacterial resistance [7] and other adverse reactions. Increasing bacterial resistance poses a major treat to human, animal health, and environment worldwide. Consequently, there is an urgent need to develop new strategy against bacterial pathogens is essential. Bacteriophage (phage) is increasingly considered as a valuable alternative to antibiotics [8]. Bacteriophages are viruses that infect bacterial cells. As one of the most abundant organisms on earth, bacteriophages play important roles in maintaining the natural abundance and distribution of microorganisms [9]. Compared to traditional antimicrobial therapy, there are many major advantages of phage therapy: high bactericidal activity, low inherent toxicity, low cost, narrow potential for including resistance. In this study, we have sequenced and analyzed the complete genome of a newly isolated ETEC K88 phage.

\section{Materials And Methods}

\section{Bacterial strains and Phage isolation}

The bacterial strains used in this study are shown in Table 1. Enterotoxigenic Escherichia coli K88 (CVCC 83902) used as a host was obtained from China Veterinary Culture Collection Center (CVCC). All strains were cultured on LB with constant shaking at $37^{\circ} \mathrm{C}$ or were stocked in LB containing $50 \%$ glycerol and stored at $-80^{\circ} \mathrm{C}$. Isolation and purification of phage vB_EcoP_E212 (referred to as E212) from farm sewage in Jilin province was done according to procedures described previously by Zhang et al[10]. 


\section{Host range investigation and efficiency of plating analysis}

The lytic capacity of phage E212 was determined by the spot test method in accordance with the ability to form lysis zone on lawn cultures of different strains [11]. Phage E212 was purified (10 $\left.\mu 1,10^{9} \mathrm{PFU} / \mathrm{mL}\right)$ and directly spotted on the surface of the bacterial lawn culture plates, and incubated overnight at $37^{\circ} \mathrm{C}$. Checked for appearance of the clear zones around the phage droplet on the plates. Efficiency of plating (EOP) was used to evaluate the ability of phage lysing bacterial strains (positive spot test). The phage titer on the host strain was calculated as $E O P=1$, and other EOPs were obtained by dividing the phage titer on the other test strains by the phage titer on the host strain.

\section{Transmission electron microscope}

Purified phage suspension $\left(10^{9} \mathrm{PFU} / \mathrm{mL}\right)$ was absorbed onto carbon coated copper grids for $10 \mathrm{~min}$, and then negatively stained with $2 \%(\mathrm{w} / \mathrm{v})$ uranyl acetate, followed by examination using a JEM-2100EX transmission electron microscope (TEM) (JEOL CO., Tokyo, Japan) [12].

\section{Phage DNA purification and sequencing}

Phage genomic DNA was extracted from a preparation with a high titer of phage particles $\left(10^{10} \mathrm{PFU} / \mathrm{mL}\right)$ using the phenol-chloroform-isoamyl alcohol method as described by Sambrook et al [13]. The DNA library was established in accordance with the protocol of the Illumina TruSeq ${ }^{\text {TM }}$ Nano DNA Sample Prep Kit. The whole genome was sequenced using the Illumina NovaSeq sequencing platform (150 bp $\times 2)$ with paired-end reads by Shanghai Personal biotechnology Co., Ltd (China) [14]. A total of $382 \mathrm{Mb}$ of sequence data were obtained with an average reading length of $150 \mathrm{bp}$. Low quality (Q-value $<20,98.51 \%$ ) reads were filtered out through Trimmomatic $v 0.36$, and approximately $98722 \times$ depth of coverage among the 25,525,764 reads. A5-MiSeq v20160825 (https://arxiv.org/abs/1401.5130) and SPAdes v3.12.0 (http://cab.spbu.ru/files/release3.12.0/manual.html) were used to assemble high quality sequencing data to constrict contig and scaffold. MUMmer v3.1 (http://mummer.sourceforge.net/) and Pilon v1.18 (https://github.com/broadinstitute/pilon) were subsequently applied to fill the remaining local inner gaps and correct single-base polymorphism for the final assembly results.

\section{Genome analysis}

Open reading frames (ORFs) were identified using the GeneMark Server (http://topaz.gatech.edu/GeneMark/genemarks.cgi) and the RAST server (http://rast.nmpdr.org/rast.cgi). The final assembled genome sequence was used to search the current protein and nucleotide databases (http://www.ncbi.nlm.nih.gov/) using the Basic Local Alignment Search Tool (BLAST).

BLASTp (https://blast.ncbi.nlm.nih.gov/Blast) was used to identify the putative functions of the encoded proteins, and BLASTn (https://blast.ncbi.nlm.nih.gov/Blast) was used to compare phage genome sequence similarity. Putative tRNA sequences were identified using tRNAscan-SE [15]. The ResFinder sever [16] (https://cge.cbs.dtu.dk/services/ResFinder/) and Virulence Factor 
Predictor [17] (https://cge.cbs.dtu.dk/services/VirulenceFinder/) were used to identify antimicrobial resistance determinates in the E212 genome.

The whole genome sequence of phage E212 was performed phylogenetic analysis with 18 published

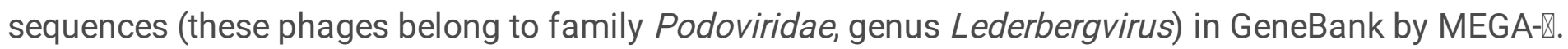
The sequence aligned by ClusrtalW, then, the maximum likelihood method was adopted to draw the phylogenetic tree. The large subunit terminase sequence was employed to determine the DNA packaging strategies of the phage. The large subunit terminase amino acid sequence obtained in this study and those of other phages were selected for multiple alignments using the Clustal W algorithm, and phylogenetic tree was constructed using MEGA7 with the neighbor-joining method. The comparative analyses of phage complete genome sequences, between E212 and the other members of the genus Lederbergvirus, were conducted using Easyfig.

\section{Results And Discussion}

One phage against Enterotoxigenic E. coli K88 (CVCC 83902) was isolated from sewage. We named this phage vB_EcoP_E212 (referred to as E212). TEM analysis revealed that E212 has an icosahedral head $(50 \pm 3 n m)$ connected to a tail $(8 \pm 2 \mathrm{~nm})$. Based on these structural features, E212 was designated as a member of Podoviridae family, Caudovirales order. (Fig.S1). Spot test and EOP results indicated that only host bacteria (CVCC 83902) was lysed by the phage E212 (Table 1).

The results of the present study showed that phage E212 has a double-stranded DNA genome with a length of 38,252 bp and an overall G + C content of $46.98 \%$. Using the RAST server, we identified 53 ORFs and predicted 44 putative protein-coding genes in the genome, 31 of which were functionally assigned. Based on bioinformatic predictions, these ORFs were categorized into four functional modules, including phage structure, host lysis, phage DNA packaging and replication and hypothetical protein (Table S2). Using tRNAscan-SE, no tRNA genes were predicted. Most of the ORFs of E212 were predicted to start with an AUG codon (47 ORFs, 88.7\%), four with GUG codon (7.5\%) and two with UUG (3.8\%). The three stop codons were present in different proportions, with UGA being the most common (31 ORFs, 58.5\%), followed by UAA (18 ORFs, 34\%) and UAG (4 ORFS, 7.5\%).

To investigate the evolutionary relationship that phage E212 within the genus Lederbergvirus, phylogentic analyses were performed on the whole genome of 18 published phage genome and E212 (Fig. 2a). In phylogram tree the phage E212 was more closely related to Escherichia phage phiv205-1, Escherichia phage JEP4 and Enterobacteria phage Sf101. The DNA packaging strategies of tailed dsDNA phages can be classified into 6 types (17 subtypes): (a) cohesive ends ( $5^{\prime} \cos$, lambda P2; 3' cos, HK97); (b) headful packaging (P2, P22, Sf6, T4, 933 W, phiPLPE, phiKZ); (c) host ends (Mu, D3112); (d) short direct terminal repeats (DTRs) (T7, N4, C-st); (e) long DTRs (SPO1); and (f) covalently bound terminal proteins (Bacillus subtilis phage $\phi 29)[18,19]$. As shown in Fig. $2 b$, the phage E212 belongs to headful packaging strategy (P22). No homologs of virulence factors (virulence genes for $E$. coli) or antimicrobial resistance genes were found in the E212 genome. However, all Lederbergvirus phages including E212 carry an integrase 
gene. Therefore, E212 is not suitable for therapy. Phages are being considered valuable alternative antimicrobial solutions, but not all phages can be considered for therapy. For safety reasons, phage genome sequence cannot carry antibiotic resistance, virulence and lysogenic genes.

A total of twenty conserved proteins are homologous (identity $>71 \%$ ) between E212 and Enterobacteria phage Sf101, among phages within the genus Lederbergvirus (Fig. 3). These include the portal, scaffold, capsid, DNA stabilization protein, head assembly protein, injection protein, tail spike, integrase, DNA methylase, DNA binding protein, i-spanin, 0 protein, holin and lysis.

\section{Conclusion}

We therefore conclude that phage E212 is a newly isolated genus Lederbergvirus.

\section{Declarations}

\section{Nucleotide sequence accession number}

The GenBank accession number for phage vB_EcoP_E212 is MZ043897.

\section{Funding}

This work was financially supported by the Agricultural Science and Technology Innovation Program of Jilin Province (CXGC2017JQ003).

\section{Compliance with ethical standards}

Conflict of interest The authors declare that they have no conflict of interest.

Ethical approval This article does not contain any studies with human participants or animals by any of the authors.

\section{References}

1. Fairbrother JM, Nadeau É, Gyles CL (2005) Escherichia coli in postweaning diarrhea in pigs: an update on bacterial types, pathogenesis, and prevention strategies. Animal Health Res Rev 6:17-39

2. Nagy B, Fekete PZ (2005) Enterotoxigenic Escherichia coli in veterinary medicine. Int J Med Microbiol 295:443-454

3. Huang GP, Li XQ, LU D et al (2018) Lysozyme improves gut performance and protects against enterotoxigenic Escherichia coli infection in neonatal piglets. Vet Res 49:20

4. Wilson RA, Francis DH (1986) Fimbriae and enterotoxins associated with Escherichia coli serogroups isolated from pigs with colibacillosis. Am J Vet Res 47:213-217 
5. Mitchell MJ, Griffiths WM, Mcewen A et al (1998) Antimicrobial drug residues in milk and meat: causes, concerns, prevalence, regulations, tests, and test performance[J]. J Food Protect 6:742-756

6. Lin SY, Huang ZH, Chen $\mathrm{HC}$ et al (2018) Multidrug-resistance Acinetobacter baumannii pneumonia in a rheumatoid arthritis patient receiving tumor necrosis factor inhibitor. Medicine 97:e11730

7. Gay N, Belmonte O, Collard J et al (2017) Review of antibiotic resistance in the Indian ocean commission: A human and animal health issue. Front Public Health 5:162

8. Carvalho C, Costa AR, Silva F et al (2017) Bacteriophages and their derivatives for the treatment and control of food-producing animal infections. Crit Rev Microbiol 43:583-601

9. Mathur MD, Vidhani S, Mehndiratta PL (2003) Bacteriophage therapy: an alternative to conventional antibiotics. JAPI 51:593-596

10. Zhang WH, Mi ZQ, Yin XY et al (2013) Characterization of Enterococcus faecalis phage IME-EF1 and its endolysin. Plos One 8:e80436

11. Jun JW, Yun SK, Kim HJ et al (2014) Characterization and complete genome sequence of a novel N4like bacteriophage, pSb-1 infecting Shigella boydii. Res Microbiol 165:671-678

12. Yuksel S, Thompson K, Ellis A et al (2001) Purification of Piscirickettsia salmonis and associated phage particles. Diseases of Aquatic Organisms 44:231-235

13. Sambrook J, Russell DW, Nina I et al (2001) Chap. 2: Bacteriophage $\lambda$ and its vectors. In: Sambrook J, Russell DW. Molecular cloning: a laboratory manual, vol 1, 3rd edn. Cold Spring Harbor, New York, pp 2.56

14. Bolger AM, Lohse M, Usadel B (2014) Trimmomatic: a flexible trimmer for Illumina sequence data. Bioinformatics 30:2114-2120

15. Schattner P, Brooks AN, Lowe TM (2005) The tRNAscan-SE, snoscan and snoGPS web servers for the detection of tRNAs and snoRNAs. Nucleic Acids Res 33:W686-W689

16. Zankari $\mathrm{E}$, Hasman $\mathrm{H}$, Cosentino $\mathrm{S}$ et al (2012) Identification of acquired antimicrobial resistance genes. J Antimicrob Chemoth 67:2640-2644

17. Joensen KG, Scheutz F, Lund $O$ et al (2014) Real-time whole-genome sequencing for routine typing, surveillance, and outbreak detection of verotoxigenic Escherichia coli. J Clin Microbiol 52:15011510

18. Casjens SR, Gilcrease EB (2009) Determining DNA packaging strategy by analysis of the termini of the chromosomes in tailed-bacteriophage virions. Methods Molecular Biology 502:91-111

19. Merrill BD, Ward AT, Grose JH et al (2016) Software-based analysis of bacteriophage genomes, physical ends, and packaging strategies. BMC Genom 17:679-694

\section{Tables}

Table 1 is not available with this version

\section{Figures}




\section{Image not available with this version}

Figure 1 
A

- MW722521.1 Salmonella phage S9-5

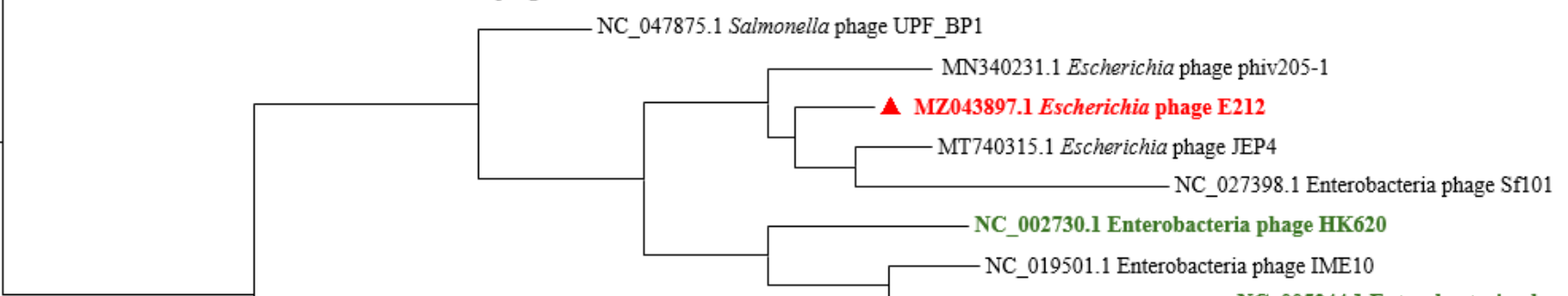

NC 005344.1 Enterobacteria phage Sf6

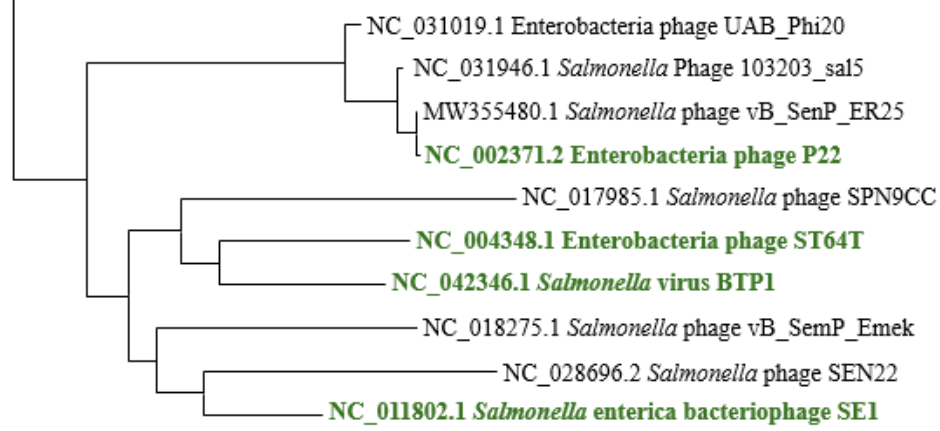

0.02

B
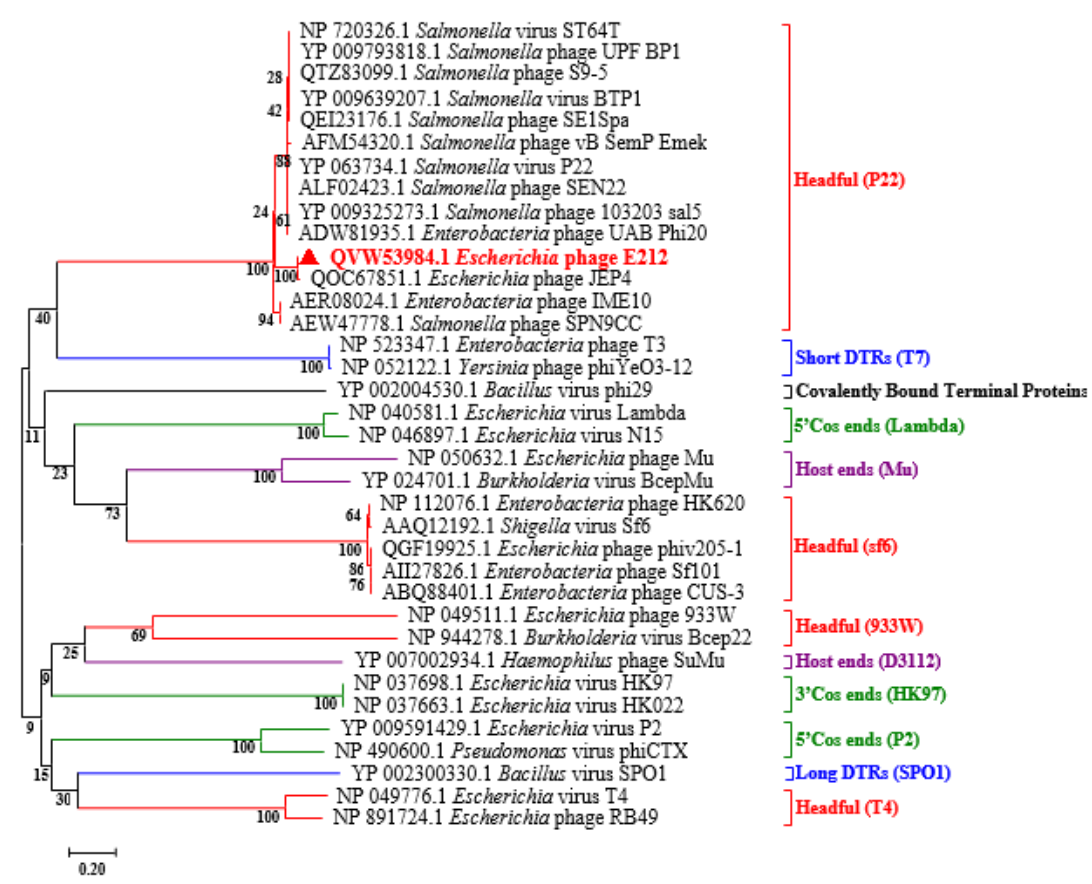

Figure 2

a: The phylogenetic tree of 19 whole genome of phages within genus Lederbergvirus b: Neighbor-joining phylogenetic tree based on amino acid sequence of terminase large subunit, showing the relationship between phage E212 and other phages. Values at the nodes indicate the bootstrap support calculated from 1000 replicates. 
Enterobacteria phage Sf101

NC_027398

Escherichia phage E212

MZ043897

Enterobacteria phage P22 NC_002371

Enterobacteria phage Sf6 NC_005344

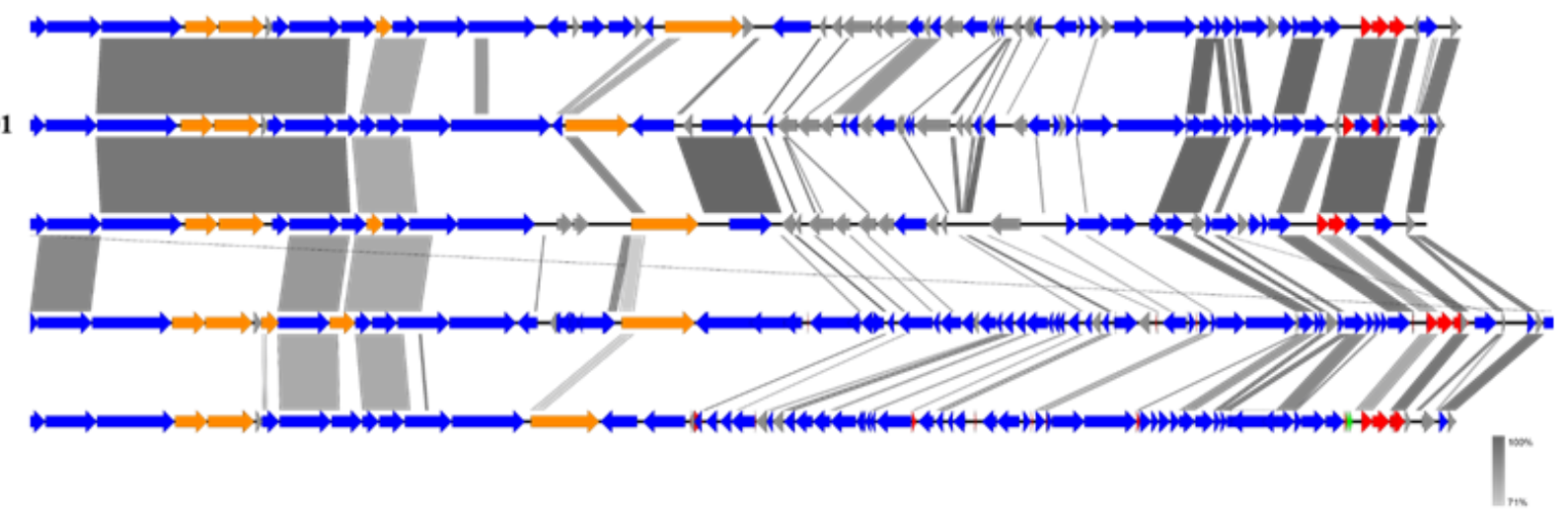
TRNA

\section{Figure 3}

Comparison of the genome of phage E212 to other Lederbergvirus members (Escherichia phage JEP4, Enterobacteria phage Sf101, Enterobacteria phage P22, Enterobacteria phage Sf6) using Easyfig software. The different color arrows represent CDS in the whole genome sequence. The direction of arrow indicated the transcription direction of each CDS. The grey bars indicated the similarity of two pairs of sequences, and the intensity of grey indicated the degree of sequence similarity.

\section{Supplementary Files}

This is a list of supplementary files associated with this preprint. Click to download.

- 4Tables.docx 